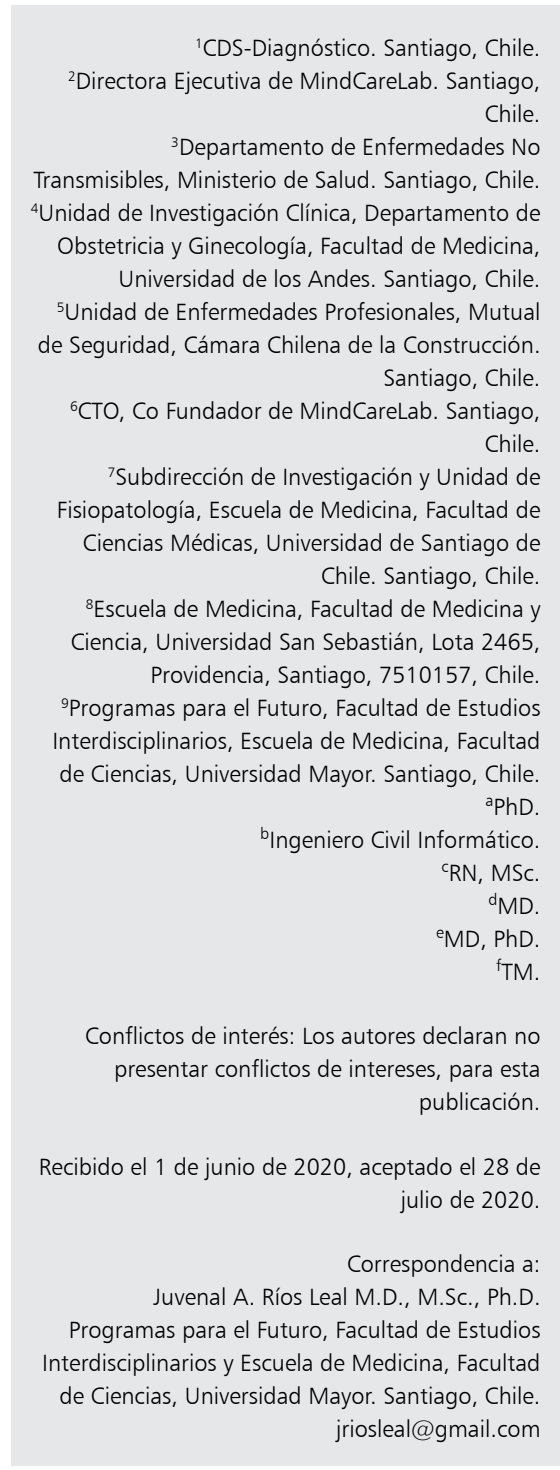

\section{Oportunidad en la salud digital: una respuesta al control de las demencias}

\author{
JUAN A. GODOY Y,a $^{1, \mathrm{a}}$ CAROLA RÍOS L. ${ }^{2, \mathrm{~b}}$, \\ PÍA VENEGAS A. ${ }^{3,4, \mathrm{c}}$, CAROLINA LÓPEZ R. ${ }^{5, \mathrm{~d}}$, \\ JOSÉ LUIS ÁLVAREZ ${ }^{6, a}$, TOMÁS P. LABBÉ ${ }^{7, e}$, \\ CAMILA RAMÍREZ M. ${ }^{1, \mathrm{f}}$, JUVENAL A. RÍOS
}

\section{Digital health services as a means to control dementia}

The increase of the elderly population with a significant load of non-communicable diseases, accelerates pathological aging and increases the risk of dementia, generating a huge health, social and economic cost for any country. Dementia does not have an effective treatment yet, therefore, the focus must remain on prevention and early diagnosis. The early stages of dementia are known as mild cognitive impairment; at this stage is still possible to mitigate the progression of the disease, however, health systems worldwide face difficulties to provide universal access to health services, due to a lack of specialists and geographical distances, interfering with the access to healthcare centers. In this scenario, WHO urged countries to implement strategies to democratize and to expand the reach of health institutions. In this document, we briefly review the global and local situation of dementias and discuss some attempts to control their progression by using revolutionary digital tools. We believe the focus should be on the population that is just beginning to show cognitive impairment.

(Rev Med Chile 2020; 148: 1018-1024)

Key words: Telemedicine; Alzheimer's Disease; Cognitive Dysfunction; Dementia.

\section{Demencias, un problema biomédico no resuelto}

4 s un hecho indiscutible que, el envejecimiento poblacional y la transformación sociodemográfica de los últimos años, son dos fenómenos que han impactado brutalmente la salud de nuestras poblaciones. Asimismo, en las personas mayores, los déficits cognitivos leves (DCL) y luego la demencia, se relacionan fuertemente con los estilos de vida (saludables o no), los que corresponden a fin de cuentas a factores de riesgo, prevenibles y/o “manejables”, según el momento del ciclo vital en que se encuentre la persona $^{1,2}$. En el último decenio, se ha acumulado abundante evidencia científica proveniente de estudios epidemiológicos, clínicos, imagenológicos, genéticos y bioquímicos que han permitido establecer una relación causal entre la diabetes mellitus 2 (DM2) y otros factores cardiometabólicos, como sustrato fisiopatológico de la Enfermedad de Alzheimer (EA) de origen esporádico ${ }^{3,4}$. Es por ello, que, el control médico y/o farmacológico, de las enfermedades no transmisibles, en especial aquellas de naturaleza metabólica, como la obesidad, 
la resistencia a la insulina y la DM2, constituirían "teóricamente" una excelente aproximación preventiva para aminorar el explosivo incremento epidemiológico de las demencias ${ }^{5,6}$. En lo farmacológico, la metformina, por más de dos décadas ha sido uno de los tratamientos orales de primera línea para la hiperglicemia y otros desbalances endocrinos, no obstante, entre sus efectos adversos más comunes, aún no superados por la industria farmacéutica, está la disminución de la absorción de vitamina $\mathrm{B} 12$ en el tracto gastrointestinal, lo que trae consigo la posibilidad de desarrollar anemia megaloblástica y trastornos asociados a disfunciones en el sistema nervioso periférico, sin mencionar los riesgos de su uso en población añosa con funcional renal deteriorada ${ }^{7-10}$.

El deterioro cognitivo leve (DCL), es un término que hace referencia a un estado mental y de reserva cognitiva que no cumple con los criterios de una demencia como tal, pero que exhibe algún grado de deterioro cerebral, distinto de un trastorno anímico o psiquiátrico clásico. Las causas más comunes de DCL están relacionadas con la edad, síntomas prodrómicos de EA y demencia vascular $(\mathrm{DVa})^{11-13}$. Cabe señalar que, está más o menos establecido la tasa de conversión de DCL a EA ${ }^{14}$, asunto que debiera tenerse en cuenta en la práctica clínica y en la planificación de una investigación clínica, cuando se necesite reportar la sensibilidad y especificidad de las pruebas que se apliquen ${ }^{11,15}$. Esto resulta fundamental en la evaluación de los presintomáticos, los que están en riesgo inminente de desarrollar EA, así como también, en aquellos con síntomas claros y manifiestos ${ }^{10,16}$. En el horizonte clínico-temporal de la demencia tipo EA, se sabe de manera muy precisa que, existe una etapa preclínica que puede empezar hasta 20 años antes del inicio de los síntomas de la enfermedad, luego una fase sintomática previa a la demencia y finalmente la demencia propiamente tal ${ }^{17-19}$.

\section{Situación epidemiólogica mundial}

Hacia el año 2011, existían 35,6 millones de personas en todo el mundo con algún tipo de demencia. En el 2015, el costo total de la demencia a nivel mundial se estimó en 818.000 millones de dólares, lo que equivale a $1,1 \%$ del producto interior bruto (PIB) mundial ${ }^{20,21}$. En relación con la Enfermedad de Alzheimer (EA), que es el tipo más común de demencia, la prevalencia entre las personas de 70-75 años es de 2-3\% y en personas de 85 años o más, aumenta dramáticamente a 20 $25 \%$, siendo discretamente superior en mujeres sobre los 85 años. En la actualidad se estima que existen unos 42 millones de pacientes con demencia y hacia el año 2030, se proyecta, alrededor de 81 millones. En el año 2050, diversos estudios epidemiológicos a nivel mundial mencionan que al menos 2.000 millones de personas serán mayores de 60 años, por lo cual, considerando que el factor de riesgo edad, se espera, un incremento dramático en el número de estos pacientes ${ }^{22,23}$.

\section{Respuesta multilateral y directrices globales}

En mayo de 2017, la asamblea de la Organización Mundial de la Salud (OMS) respaldó un Plan de Acción en relación con una respuesta sanitaria global y coordinada para controlar el preocupante avance de la demencias ${ }^{24}$. Este plan de la OMS, junto a otros organismo internacionales, regionales y nacionales, establece los siguientes lineamientos generales; i) reconocimiento de la demencia como prioridad para los sistemas públicos de salud del planeta; ii) concientización y puesta en marcha de iniciativas para satisfacer las necesidades de la población afectada; iii) fortalecimiento de la prevención, diagnóstico precoz, tratamiento, levantamiento de sistemas de información, registro y vigilancia epidemiológica, apoyo a los cuidadores e investigación e innovación.

\section{Situacional sanitaria local}

En Chile las personas mayores representan $19,32 \%$ de la población, lo que es equivalente a 3.439.599 adultos mayores, en particular, los adultos mayores de la cuarta edad, es decir de 80 años y más, ascienden a 573.271 personas, lo que representa 3,22\% del total de la población ${ }^{25}$. Un estudio nacional realizado por el Servicio Nacional del Adulto Mayor, (SENAMA), en el año 2009, estimó que en el país, alrededor de 1,06\% de la población, presenta algún tipo de demencia, en las personas de más de 60 años, alcanza 7,1\%, y este aumento es mayor hacia los 75 años, alcanzando $13 \%$ y en los mayores de 85 años, $36,2 \%{ }^{26,27}$. En tanto, los estudios de la Corporación Profesional 
de Alzheimer y otras demencias (COPRAD), reportan alrededor de 200 mil pacientes con EA, una cifra que podría triplicarse para el $2050^{27}$. A esto se debe agregar el enorme costo anual que significa para las familias que cuidan estos enfermos y que trae consigo las inequidades propias de nuestro sistema que aún está en vías de maduración ${ }^{28,29}$. Ante esta realidad, el Ministerio de Salud de Chile (MINSAL), ha desplegado desde el 2017 un Plan Nacional de Demencias, instrumento que guía las acciones preventivas, de diagnóstico precoz, tratamiento y rehabilitación de este problema de salud (PS). Adicionalmente a este esfuerzo estatal, se materializa a partir de Octubre 2019, la "Enfermedad de Alzheimer y otras demencias", como un nuevo problema GES (Garantías Explícitas en Salud) lo que se traduce en otorgar a la población afectada, garantías legales de oportunidad diagnostica, tratamiento y seguimiento, con plazos y prestaciones definidas ${ }^{30}$.

\section{Salud digital al rescate de la salud mental}

\section{Situación de la salud digital desde la OMS}

Las iniciativas de salud digital están progresivamente en funcionamiento, en la practica $83 \%$ de los Estados Miembros de la OMS ya comenzaron su implementación, y 90\% de ellas tienen una estrategia de salud "en línea"31,32. Sin embargo, las diferentes formas de salud digital y tecnología presentan diferentes modalidades a nivel mundial. La telemedicina se difunde más ampliamente que los registros electrónicos de salud, que se usan más comúnmente que los grandes datos en entornos de atención médica. Pero es importante destacar que a pesar de que la salud digital representa una realidad global, se adopta e implementa de manera diferente en todo el mundo ${ }^{32,33}$.

\section{Salud digital, algunos conceptos claves}

La salud digital es un concepto más amplio que solo la telemedicina. Según la Administración de Drogas y Alimentos de los Estados Unidos (FDA) la salud digital, incluye categorías como; salud móvil (mHealth), tecnología de información de salud (IT), dispositivos portátiles, telesalud y telemedicina ${ }^{34}$.

En términos generales, implica conectar datos relacionados con la salud, incluyendo los datos generados por los propios pacientes, y aprovechar el potencial médico de las herramientas tecnológicas de uso común, como teléfonos inteligentes, aplicaciones, redes sociales y dispositivos de detección ubicados en nuestro entorno. La mayoría de estas herramientas no están concebidas inicialmente para uso médico y no se comercializaban como dispositivos médicos, pero han alcanzado ese $\mathrm{rol}^{32}$.

Se reconoce la necesidad de una asociación entre los sistemas humanos y digitales para lograr un cambio en la atención médica, por ejemplo, la tecnología para apoyar mejor a los trabajadores sobrecargados en la atención primaria entre otras situaciones ${ }^{35}$.

La innovación en salud digital también enfrenta varios desafíos éticos y políticos. Para que los desarrollos de salud digital produzcan innovación e impactos en la salud, ya sea a nivel individual o de las poblaciones, deben cumplir condiciones ${ }^{32}$. Por ejemplo, los datos son de suma importancia, el acceso a cantidades suficientes, por tanto, es una condición primaria para el desarrollo de herramientas innovadoras de diagnóstico, terapéuticas y de monitoreo en esta área. En segundo lugar, lograr una sinergia con las realidades legales existentes en materia de protección de datos, seguridad de datos y privacidad son clave para la innovación en salud digital. Por otro lado, es importante desarrollar marcos de responsabilidad en el contexto del uso y el manejo de información tanto para comunicar o compartir información de salud para los involucrados. En cuarto lugar, la seguridad y eficacia juegan un rol preponderante, cada una de las aplicaciones en salud digital deberá responder a los más altos estándares para otorgar la confianza tanto al individuo como al entorno ${ }^{32,35}$.

\section{Demencias y algunas soluciones digitales}

En el contexto de la salud mental, si se le compara con otras áreas de la neuropsiquiatría tanto a nivel mundial como en el medio local, como es el caso del Grupo de la Dra. Graciela Rojas en la Universidad de Chile ${ }^{36-40}$, la salud digital ha tenido un lento pero sostenido desarrollo en el ámbito de las Demencias. Así, los dispositivos móviles, tabletas, relojes inteligentes, trajes con sensores incluidos $\mathrm{y}$ aplicaciones (APP), constituyen un conjunto de herramientas tecnológicas que nos ofrecen una gran oportunidad para apoyar el diagnóstico precoz y monitoreo remoto de una población que envejece rápidamente ${ }^{41,42}$. A la fecha existen pocas innovaciones en el ámbito de la detección de DCL, 
ya que la mayoría de los desarrollos han estado enfocados en medir parámetros de la enfermedad avanzada.

En este sentido, el 2019 se publicó una revisión sistemática ${ }^{43}$, donde se incluyeron 26 estudios primarios, para responder preguntas como ¿Cuál es el uso real de las tecnologías en el hogar, para la detección temprana y seguimiento del deterioro cognitivo leve? Las principales experiencias que "hipotéticamente" responden a esta pregunta, fueron sistematizadas en los 4 siguientes grupos de soluciones tecnológicas, validadas internacionalmente:

i) Sensores fijos integrado-pasivos en la casa o en el auto; se utilizaron sensores de movimiento dentro de la casa, para evaluar velocidad de los pasos, que da cuenta de la marcha y otros parámetros como contabilizar el número movimientos nocturnos, que da cuenta indirectamente de la calidad del sueño, en la mayoría había diferencias significativas al comparar sanos con pacientes DCL $(+)$.

ii) Sensores portátiles basados en localización (GPS): en este grupo los sensores están instalados en los celulares u otro aparato que se movilice con el paciente, de modo que pueda dar cuenta de su recorrido fuera de la casa, la posibilidad de pérdida por olvido de la ruta de vuelta a la casa, además de generar alarmas a los familiares. La mayoría de estos mostró diferencias significativas entre DCL y controles.

iii) Soluciones tecnológicas dedicadas o intencionadas en formato APP; en este grupo, se evaluó el rendimiento de las personas en encuestas o juegos en teléfonos móviles inteligentes, a través de aplicaciones diseñadas para estos fines, lo que en sí mismo ya en un desafío por la barrera de “alfabetización digital del adulto mayor". La gran mayoría de los estudios mostró buena correlación con el diagnóstico de DCL versus controles.

iv) Uso de soluciones tecnológicas no diseñadas para esto fines: en este caso los datos provienen de parámetros secundarios derivados de los usos de una tecnología "X", por ejemplo, el movimiento del "mouse", la vigilancia remota de cómo y cuánto usa el PC un adulto mayor, entre otras. Este grupo, tuvo muchas limitaciones de análisis, sesgos y no fue posible extraer conclusiones precisas.
Desde el punto de vista de la gestión asistencial, el uso de estas herramientas, debiera invadir los tres niveles de atención e incluso el nivel central como ya se está haciendo, de modo de dotar de infraestructura digital y recurso humano entrenado en utilizarla, en las siguientes instancias que tengan a su cargo, la población adulta y adulta mayor; Corporaciones Municipales, Centros de Atención Primaria (CESFAM, COSAM), Hospitales Públicos, Clínicas Privadas, Servicio Nacional del Adulto Mayor (SENAMA) y Hospital Digital del Ministerio de Salud.

\section{Conclusiones}

Es muy evidente que la situación epidemiológica mundial y local aquí presentada, en relación con las demencias, nos debe hacer buscar intensamente, métodos innovadores para el control de su desbordado crecimiento.

A esto se suma, el complejo escenario sanitario que vivimos secundario a la pandemia por el virus SARS-CoV2 que origina la "COVID19", y que por un lado, ya sabe, tendrá consecuencias catastróficas en relación con el control de las enfermedades crónicas no transmisibles (hipertensión, diabetes, cáncer y demencias) ${ }^{44,45}$, pero, por otro, la presión biológica-social de la crisis, nos ha obligado a consolidar, entre otras cosas, procesos de revolución sanitaria de manera definitiva, como lo es "la salud digital".

En ese contexto el adulto y el adulto mayor con problemas iniciales de salud mental de cualquier parte del mundo tendrá menores posibilidades de acceder a atención médica presencial oportuna y mucho menos a una evaluación temprana por un especialista.

Por ello, el desarrollo de la salud digital dirigido y hecho a la medida de un problema específico de salud, ocupado en una ventana terapéutica razonable y no utilizado como un fin, sino como un medio complementario a la medicina moderna, será esencial para enfrentar los desafíos sanitarios de un mundo en donde las enfermedades crónicas no infecciosas ahora se verán también afectadas por los vaivenes de las "infecciosas", las cuales, erróneamente, ya creíamos casi derrotadas.

Agradecimientos: El autor correspondiente (JAR) agradece a la Escuela de Medicina, de la 
Facultad de Ciencias y a la Oficina de Programas para el Futuro, de la Facultad de Estudios Interdisciplinarios, ambas instancias de la Universidad Mayor de Chile, por acoger positivamente el desarrollo de la Salud Digital como un área nueva, dentro de una futura innovación curricular, tanto para el pregrado como para el posgrado de esta Casa de Estudios Superiores. Los autores Carola Ríos y José Luis Álvarez agradecen especialmente a CORFO, al Comité de Transformación Digital, a los Centros de Pilotaje de Innovación en Salud (CePIS), a la Asociación de Municipalidades de Chile y a la Asociación Chilena de Farmacias Populares (ACHIFARP), por el apoyo constante en el desarrollo de la solución MindCareLab.

\section{Referencias}

1. Kivipelto M, Mangialasche F, Ngandu T. Lifestyle interventions to prevent cognitive impairment, dementia and Alzheimer disease. Vol. 14, Nature Reviews Neurology. Nature Publishing Group; 2018. p. 653-66.

2. Peters R, Booth A, Rockwood K, Peters J, D’Este C, Anstey KJ. Combining modifiable risk factors and risk of dementia: A systematic review and meta-analysis. Vol. 9, BMJ Open. BMJ Publishing Group; 2019. p. e022846.

3. Beydoun MA, Beydoun HA, Wang Y. Obesity and central obesity as risk factors for incident dementia and its subtypes: A systematic review and meta-analysis. Vol. 9, Obesity Reviews. NIH Public Access; 2008. p. 204-18.

4. Ríos JA, Cisternas P, Arrese M, Barja S, Inestrosa NC. Is Alzheimer's disease related to metabolic syndrome? A Wnt signaling conundrum. Prog Neurobiol 2014; 121.

5. Ngandu T, Lehtisalo J, Solomon A, Levälahti E, Ahtiluoto S, Antikainen R, et al. A 2 year multidomain intervention of diet, exercise, cognitive training, and vascular risk monitoring versus control to prevent cognitive decline in at-risk elderly people (FINGER): A randomised controlled trial. Lancet 2015; 385 (9984): 2255-63.

6. Rosenberg A, Ngandu T, Rusanen M, Antikainen R, Bäckman L, Havulinna S, et al. Multidomain lifestyle intervention benefits a large elderly population at risk for cognitive decline and dementia regardless of baseline characteristics: The FINGER trial. Alzheimer's Dement 2018; 14 (3): 263-70.

7. Metformin and Risk of Alzheimer's Disease Among Community-Dwelling People With Diabetes: A National Case-Control Study | The Journal of Clinical Endocrinology \& Metabolism | Oxford Academic.
8. Kim J, Ahn CW, Fang S, Lee HS, Park JS. Association between metformin dose and vitamin B12 deficiency in patients with type 2 diabetes. Medicine (Baltimore) 2019; 98 (46): e17918.

9. Davis JC, Verhagen E, Bryan S, Liu-Ambrose T, Borland J, Buchner D, et al. 2014 consensus statement from the first economics of physical inactivity consensus (EPIC) conference (Vancouver). In: British Journal of Sports Medicine. BMJ Publishing Group; 2014. p. 947-51.

10. Morley JE, Morris JC, Berg-Weger M, Borson S, Carpenter BD, del Campo N, et al. Brain Health: The Importance of Recognizing Cognitive Impairment: An IAGG Consensus Conference. J Am Med Dir Assoc 2015; 16 (9): 731-9.

11. Petersen RC, Smith GE, Waring SC, Ivnik RJ, Tangalos EG, Kokmen E. Mild cognitive impairment: Clinical characterization and outcome. Arch Neurol 1999; 56 (3): 303-8.

12. Petersen RC, Doody R, Kurz A, Mohs RC, Morris JC, Rabins PV, et al. Current concepts in mild cognitive impairment. Arch Neurol 2001; 58 (12): 1985-92.

13. Morris JC, Storandt M, Miller JP, McKeel DW, Price JL, Rubin EH, et al. Mild cognitive impairment represents early-stage Alzheimer disease. Arch Neurol 2001; 58 (3): 397-405.

14. Lu X, Chen J, Shu H, Wang Z, Shi Y, Yuan Y, et al. Predicting conversion to Alzheimer's disease among individual high-risk patients using the Characterizing AD Risk Events index model. CNS Neurosci Ther 2020 Apr.

15. Luis CA, Barker WW, Loewenstein DA, Crum TA, Rogaeva E, Kawarai T, et al. Conversion to dementia among two groups with cognitive impairment: A preliminary report. Dement Geriatr Cogn Disord 2004; 18 (3-4): 307-13.

16. Borson S, Frank L, Bayley PJ, Boustani M, Dean M, Lin PJ, et al. Improving dementia care: The role of screening and detection of cognitive impairment. Vol. 9, Alzheimer's and Dementia. Elsevier Inc.; 2013. p. 151-9.

17. Malek-Ahmadi M, Pérez SE, Chen K, Mufson EJ. Braak stage, cerebral amyloid angiopathy, and cognitive decline in early Alzheimer's disease. J Alzheimer's Dis 2020; 74 (1): 189-97.

18. Dubois B, Hampel H, Feldman HH, Scheltens P, Aisen $\mathrm{P}$, Andrieu S, et al. Preclinical Alzheimer's disease: Definition, natural history, and diagnostic criteria. Vol. 12, Alzheimer's and Dementia. Elsevier Inc.; 2016. p. 292323.

19. Khan TK. An algorithm for preclinical diagnosis of Alzheimer's disease. Vol. 12, Frontiers in Neuroscience. Frontiers Media S.A.; 2018. 
20. Scheltens P, Blennow K, Breteler MMB, de Strooper B, Frisoni GB, Salloway S, et al. Alzheimer's disease. Lancet [Internet] 2016; 388 (10043): 505-17. Available from: https:/www.sciencedirect.com/science/article/pii/ S0140673615011241?via\%3Dihub [citado el 16 de abril de 2019].

21. 2016 Alzheimer's disease facts and figures. Alzheimer's Dement 2016; 12 (4): 459-509.

22. Ferri CP, Prince M, Brayne C, Brodaty H, Fratiglioni L, Ganguli M, et al. Global prevalence of dementia: A Delphi consensus study. Lancet 2005; 366 (9503): 2112-7.

23. Barrera López FJ, López Beltrán EA, Baldivieso Hurtado N, Maple Álvarez IV, López Moraila MA, Murillo Bonilla LM, et al. Diagnóstico Actual de la Enfermedad de Alzheimer. Rev Med Clínica 2018; 2 (2): 57-73.

24. World Health Organization. Global action plan on the public health response to dementia 2017 - 2025 [Internet]. Geneva: World Health Organization. 2017 [citado el 29 de mayo de 2020]. Available from: http:// www.who.int/mental_health/neurology/dementia/ action_plan_2017_2025/en/

25. Encuesta Casen - Observatorio Social - Ministerio de Desarrollo Social.

26. MIDESO - Senama - Sociedad de Geriatría - Minsal: Subsecretaría de Salud Pública, División de Prevención y Control De Enfermedades. Departamento De Ciclo Vital.

27. Slachevsky A. AP. MJ. \& RR. Enfermedad de Alzheimer y otras demencias en Chile: Propuesta de un Plan Nacional de Alzheimer y otras demencias. Chile: Corporación Profesional de Alzheimer y otras demencias (Coprad); Sociedad de Neurología, Neuropsicología y Neurocirugía de Chile. Sone. 2012.

28. Hojman DA, Duarte F, Ruiz-Tagle J, Budnich M, Delgado C, Slachevsky A. The cost of dementia in an unequal country: The case of Chile. Federici S, editor. PLoS One [Internet]. 2017; 12 (3): e0172204. Available from: https://dx.plos.org/10.1371/journal.pone.0172204 [citado el 30 de mayo de 2020].

29. Custodio N, Wheelock A, Thumala D, Slachevsky A. Dementia in Latin America: Epidemiological Evidence and Implications for Public Policy. Front Aging Neurosci [Internet] 2017; 9: 221. Available from: http://journal. frontiersin.org/article/10.3389/fnagi.2017.00221/full [citado el 30 de mayo de 2020].

30. Enfermedad de Alzheimer y otras demencias - Orientación en Salud. Superintendencia de Salud, Gobierno de Chile. [Internet]. Available from: http://www.supersalud.gob.cl/difusion/665/w3-printer-18653.html [citado el 29 de mayo de 2020].
31. Alami H, Gagnon M-P, Fortin J-P. Digital health and the challenge of health systems transformation. mHealth 2017; 3: 31-31.

32. Vayena E, Haeusermann T, Adjekum A, Blasimme A. Digital health: meeting the ethical and policy challenges. Vol. 148, Swiss medical weekly. EMH Media; 2018. p. w14571.

33. World Health Organization (WHO). WHO guideline: recommendations on digital interventions for health system strengthening: evidence and recommendations [Internet]. Vol. WHO/RHR/19, Documentos Técnicos. World Health Organization; 2019. 13 p. Available from: https://www.ncbi.nlm.nih.gov/books/NBK541902/ [citado el 29 de mayo de 2020].

34. Garvey W, Garber A, Mechanick J, Bray G, Dagogo-Jack S, Einhorn D, et al. American Association of Clinical Endocrinologists and American College of Endocrinology Position Statement on the 2014 Advanced Framework for a New Diagnosis of Obesity as a Chronic Disease. Endocr Pract 2014; 20 (9): 977-89.

35. The Lancet Digital Health.

36. Eysenbach G. What is e-health? Vol. 3, Journal of Medical Internet Research. Journal of Medical Internet Research; 2001. p. 1-5.

37. Castro A, Larrain A, Fritsch R, Rojas G. Telepsiquiatría: Una revisión sistemática cualitativa. Rev Med Chile 2012; 140 (6): 789-96.

38. Rojas G, Martínez V, Martínez P, Franco P, Jiménez-Molina Á. Improving Mental Health Care in Developing Countries Through Digital Technologies: A Mini Narrative Review of the Chilean Case. Front Public Heal [Internet] 2019; 7: 391. Available from: https://www. frontiersin.org/article/10.3389/fpubh.2019.00391/full [citado el 29 de mayo de 2020].

39. Rojas G, Guajardo V, Martínez P, Castro A, Fritsch R, Moessner $\mathrm{M}$, et al. A remote collaborative care program for patients with depression living in rural areas: Open-label trial. J Med Internet Res 2018; 20 (4).

40. Martínez V, Rojas G, Martínez P, Gaete J, Zitko P, Vöhringer PA, et al. Computer-Assisted Cognitive-Behavioral Therapy to Treat Adolescents With Depression in Primary Health Care Centers in Santiago, Chile: A Randomized Controlled Trial. Front Psychiatry [Internet] 2019; 10: 552. Available from: https://www. frontiersin.org/article/10.3389/fpsyt.2019.00552/full [citado el 29 de mayo de 2020].

41. Dorsey ER, Papapetropoulos S, Xiong M, Kieburtz K. The First Frontier: Digital Biomarkers for Neurodegenerative Disorders. Digit Biomarkers 2017; 1 (1): 6-13.

42. Doraiswamy PM, Narayan VA, Manji HK. Mobile and 
pervasive computing technologies and the future of Alzheimer's clinical trials. NPJ Digit Med 2018; 1 (1): 1-4.

43. Piau A, Wild K, Mattek N, Kaye J. Current State of Digital Biomarker Technologies for Real-Life, Home-Based Monitoring of Cognitive Function for Mild Cognitive Impairment to Mild Alzheimer Disease and Implications for Clinical Care: Systematic Review. J Med Internet Res [Internet] 2019; 21 (8): e12785. Available from: http://www.ncbi.nlm.nih.gov/pubmed/31471958 [citado el 29 de mayo de 2020].

44. Wang H, Li T, Barbarino P, Gauthier S, Brodaty H, Molinuevo JL, et al. Dementia care during COVID-19. Vol. 395, The Lancet. Lancet Publishing Group; 2020. p. 1190-1.

45. Pal R, Bhadada SK. COVID-19 and non-communicable diseases. Postgraduate Medical Journal. BMJ Publishing Group; 2020. 